\title{
Regulatory and Commercial Obsolescence Risks of Materials and Processes
}

\author{
Paavo Heiskanen, Premysl Janik, Oliver Reiff-Musgrove, Mikko Nikulainen and Tim \\ Becker*
}

This article summarises the current challenges related to legal obligations and restrictions associated with the use of chemicals, directly affecting space industrial sector. It also highlights issues surrounding the obsolescence of materials and processes, which are indirectly linked to the restrictions in use, but often have a serious impact on the space industry. Methods for risk assessment and means for the mitigation of risks associated with material and process obsolescence are described in the article. The most relevant substances under restrictions are discussed in detail. Lastly, the evolution in waste management, as well as some implications of the UK withdrawal from the EU are additionally analysed.

\section{Introduction}

Health, safety and environmental regulations and directives such as REACH, CLP and RoHS affect the future availability of space components, materials, manufacturing processes and related technologies. Space programmes are exposed to regulatory obsolescence risk due to legal obligations restricting or banning the use of hazardous substances. Furthermore, commercial obsolescence risks are evident as larger business sectors may drive the evolution of key markets to their needs, which are not necessarily fit for purpose for space applications. The issue impacts space industry competitiveness due to higher costs of new product qualifications, coupled with a weaker heritage position. These regulations are very de-

DOI: $10.21552 / \mathrm{icrl} / 2020 / 1 / 4$

* Paavo Heiskanen, former ESA REACH officer, Technical Reliability and Quality Division (TEC-QE), European Space Agency, for correspondence: <reach.officer@esa.int>. Premysl Janik, present ESA REACH Officer, Technical Reliability and Quality Division (TEC-OE), European Space Agency,for correspondence: <premysl.janik @esa.int>. Oliver Reiff-Musgrove, Young Graduate Trainee, Technical Reliability and Quality Division (TEC-QE), European Space Agency, for correspondence: <oliver.musgrove@esa.int>. Mikko Nikulainen, Head of Technical Reliability and Quality Division (TEC-QE), European Space Agency, for correspondence: <mikko.nikulainen@esa.int>. Tim Becker, Senior Legal Advisor, REACHLaw Ltd, for correspondence: $<$ tim .becker@reachlaw.fi>. The presented paper was supported by ESA funding, contracts: CTR\#4000106152 and CTR\#4000112903 (CCN1).

1 Regulation (EC) No 1907/2006 of the European Parliament and of the Council of 18 December 2006 concerning the Registration, Evaluation, Authorisation and Restriction of Chemicals (REACH). sirable and ambitious, contributing to a safer and healthier environment and support transfer to a more circular economy and non-toxic environment. However, they cause engineering and management challenges in highly regulated niche markets such as space. Managing these risks correctly will greatly increase the likelihood of project success and business sustainability.

\section{Legal Background}

\section{REACH and CLP Regulations}

All substances manufactured in or imported to the EU/EEA (European Economic Area) in quantities of one tonne or more per year require a $\mathrm{REACH}^{1}$ registration for continued placing on the market, unless specific exemptions apply. The registration authority is the European Chemicals Agency (ECHA). The final registration deadline for existing substances passed on 31 May 2018. In total, more than 20000 substances have been registered to date, as part of approximately 100 ooo registrations. Where more information is needed to confirm the risk to human health or the environment, the registered substances are evaluated with respect to their physico-chemical and toxicological properties.

Authorisation and restriction requirements are applied to substances identified as being of particular concern. So-called Substances of Very High Concern (SVHC) are placed on the candidate list for au- 


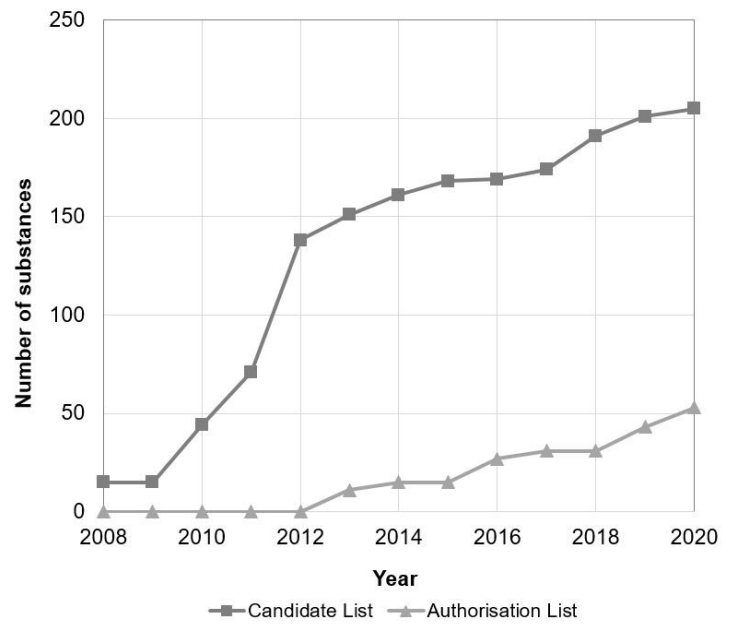

Figure 1: Evolution of the number of substances on REACH candidate and authorisation lists (Source: ECHA)

thorisation. Safe use information regarding such SVHCs present in articles above $0.1 \%$ weight-byweight $(\mathrm{w} / \mathrm{w})$ must be communicated in the supply chain by EU/EEA article suppliers under REACH Article 33 .

Currently there are 54 substances in the authorisation list (Annex XIV) of the REACH regulation, which mandates that for continued use after a sunset date an authorisation is required, unless a specific exemption applies. The latest update of the authorisation list took place through Commission Regulation (EU) 2020/171 of 6 February 2020. The candidate list for authorisation currently contains 205 substances and is updated biannually, with the last update having taken place on 16 January 2020. Fig. 1 shows the evolution of the numbers of substances within these regulatory lists over time.

Restrictions are included in REACH Annex XVII for hazardous substances (also other than SVHCs) when there is an unacceptable risk to human health or the environment, arising from their manufacture, use or placing on the market, which needs to be addressed on an EU-wide basis. Restriction means any condition for, or prohibition of, the manufacture, use or placing on the market. Restrictions need to be complied with by industry, as it is not possible to apply for authorisation to use within such restriction. However, derogations and extended use timelines for certain critical applications may be foreseen in the conditions of the restriction. Furthermore, some gener- al exemptions from restrictions apply, such as for scientific research and development.

The CLP ${ }^{2}$ Regulation (EC) No 1272/2008 complements REACH by setting out rules for the classification, labelling and packaging of substances and mixtures. These rules follow the United Nations' Globally Harmonised System of Classification and Labelling of Chemicals (UN GHS). Annex VI of CLP establishes an evolving list of substances with a legally binding harmonised classification and labelling. If a substance meets the criteria for classification in the hazard class carcinogenicity, germ cell mutagenicity or reproductive toxicity (CMR) category $1 \mathrm{~A}$ or $1 \mathrm{~B}$, it shall normally be subject to such harmonised classification and fulfils the REACH definition of an SVHC. Thus, it will be eligible for inclusion in the REACH candidate list for authorisation.

ECHA's Integrated Regulatory Strategy (IRS) ${ }^{3}$ brings together the various regulatory processes. Its Public Activities Coordination Tool (PACT) ${ }^{4}$ displays the substances under regulatory scrutiny. In December 2019, ECHA has also published the 'universe of registered substances', indicating the general regulatory roadmap for each registered substance (chemical universe list). These strategies and tools are useful monitoring instruments for industry.

\section{RoHS Directive}

The RoHS ${ }^{6}$ Directive governs specific restrictions of the use of certain hazardous substances in electrical and electronic equipment, for example lead metal. RoHS does not directly affect space systems, as it has clear exclusions from its scope for a number of cases, with 'equipment designed to be sent into space' being one of them. However, it may affect ground systems and other facilities, such as launch sites and ground stations, unless other general exclusions

2 Regulation (EC) No 1272/2008 of the European Parliament and of the Council of 16 December 2008 on classification, labelling and packaging of substances and mixtures (CLP).

3 See $<$ https://echa.europa.eu/substances-of-potential-concern $>$ accessed 13 May 2020.

4 See <https://echa.europa.eu/pact> accessed 13 May 2020.

5 See $<$ https://echa.europa.eu/universe-of-registered-substances $>$ accessed 13 May 2020.

6 Directive 2011/65/EU of the European Parliament and of the Council of 8 June 2011 on the restriction of the use of certain hazardous substances in electrical and electronic equipment. 
from the scope or specific exemptions can be established. However, as more and more satellites use subsystems that consist in part or fully of Commercial Off The Shelf (COTS) electronics components and surface mount assemblies, this directive also in effect limits the available market for space use. In the frame of the ongoing RoHS review, the space industry has communicated to the European Commission that the existing exclusions from the RoHS scope covering the space sector are essential for the continued availability of reliable electronics. Also, if new substances should be included in the list of restricted substances under RoHS, it may affect space use due to commercial obsolescence as mentioned above.

\section{Waste Framework Directive (as revised)}

The recent revision of the Waste Framework Directive 'WFD" introduces a new specific obligation for article suppliers in the EU (and potentially EEA), which will apply starting from 5 January 2021. ${ }^{8}$ According to Article 9(1)(i) of the revised WFD these actors have to communicate safe use information relating to SVHCs in their supplied articles and complex objects to ECHA, in line with the supply chain communication obligation under REACH Article 33 mentioned above. ECHA is currently establishing a database for Substances of Concern in Products (SCIP) to collect the information received from industry and provide access to it, primarily to waste operators.

7 Directive (EU) 2018/851 of the European Parliament and of the Council of 30 May 2018 amending directive 2008/98/EC on waste

8 The need for a possible postponement of the deadline due to the impacts of the COVID-19 situation is currently being reviewed by the European Commission (DG ENV), based on various stakeholder requests.

9 Council Directive 89/391/EEC of 12 June 1989 on the introduction of measures to encourage improvements in the safety and health of workers at work [1989] OJ L 183, 1-8

10 Council Directive 98/24/EC of 7 April 1998 on the protection of the health and safety of workers from the risks related to chemical agents at work [1998] OJ L 131, 11-23

11 Directive 2004/37/EC of the European Parliament and of the Council of 29 April 2004 on the protection of workers from the risks related to exposure to carcinogens or mutagens at work [2004] OJ L 158, 23-34

12 IEC 62402:2019 - Obsolescence management [2019]

13 ECSS-E-AS-11C - Adoption Notice of ISO 16290, Space systems Definition of the Technology Readiness Levels (TRLs) and their criteria of assessment [2014]
Member States have to transpose the revised WFD into their national laws by 5 July 2020.

\section{OSH Directives}

Chemical safety at work in the EU is regulated by three key directives: the Occupational Safety and Health Framework Directive $(\mathrm{OSH})^{9}$, the Chemical Agents Directive $(\mathrm{CAD})^{10}$ and the Carcinogens and Mutagens Directive $(\mathrm{CMD})^{11}$. These directives set minimum standards to ensure the safety and health of workers at work. CAD introduces specific rules to protect workers from the risks related to chemical agents at work, and the CMD introduces specific requirements for carcinogenic and mutagenic substances. These directives provide the basis for the setting of EU-wide Occupational Exposure Limits (OELs) for defined hazardous substances. Since the beginning of 2019, ECHA has been tasked to provide scientific recommendations on these limits. OELs may be defined to be measured from the workplace air, unless otherwise specified. In addition, Biological Limit Values (BLV) may be foreseen under CAD. For a practical example on how OSH directives are affecting the space industry see paragraph 5.3 on lead metal.

\section{Obsolescence}

As defined in the International Electrotechnical Commission (IEC) standard 62402:2019 ${ }^{12}$, obsolescence is the transition of an item from available to unavailable from the manufacturer, in accordance with the original specification. Chemical legislation has caused instances in the European space industry where a material or process has become obsolete. This causes difficult, expensive and resource intensive issues for the industry due to needs for requalification, delays, project risks and their mitigation. In many cases substitution alternatives exist, but their technical maturity and suitability is often very diverse. The metric for assessing the technological maturity of replacements is defined in terms of Technology Readiness Levels (TRL) ${ }^{13}$.

Obsolescence can be divided into two categories: regulatory and commercial obsolescence. Regulatory obsolescence means that a material or process has become unavailable to the industry due regulatory 


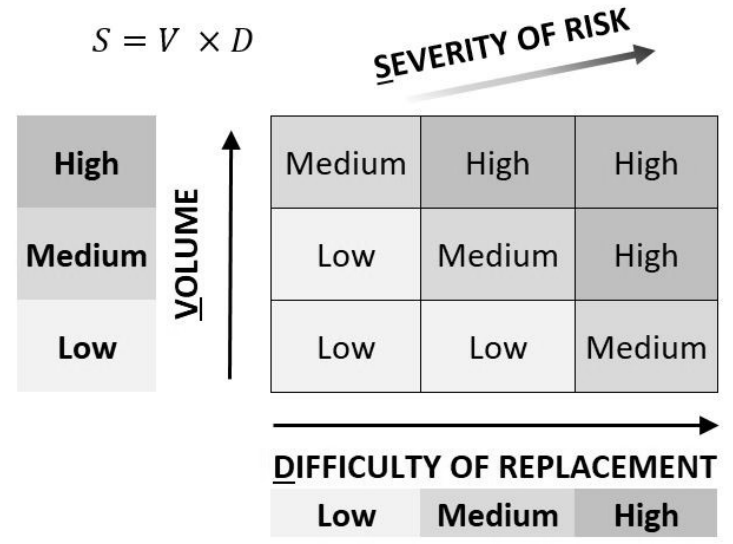

Figure 2: Severity Metric

incompatibility, ie a specific coating process can no longer be performed as the required chemical substance is no longer permitted. Commercial obsolescence dictates that a material or process has become unavailable to the industry as a consequence of no longer being offered by the market for commercial reasons. For example, this can be due to the excessive costs of fulfilling the regulatory requirements, a shrinking market due to strict authorisation of use, the negative image for using a hazardous chemical, or other reasons. The space industry has witnessed critical chemicals becoming obsolete in the span of a few years from the first indication that it has become under scrutiny by the regulators. This time scale is incompatible with common project durations in the space domain.

\section{Risk Assessment and Mitigation}

The European Cooperation for Space Standardization (ECSS) $)^{14}$ uses the industry standard definition of risk consisting of two components: likelihood of occurrence and impact of the negative consequence (severity). In the context of environmental regulation causing risks to projects, we define a quantitative quantized metric for the severity of the risk in terms of volume of use and ease of replacement as shown in Eq. 1.

$\mathrm{S}=\mathrm{V} \times \mathbf{D} \quad(1)$

Where $\mathrm{S}$ is severity, $\mathrm{V}$ is the volume of use and $\mathrm{D}$ is the difficulty of replacement. The parameters are defined as follows, and the resulting metric is shown in Fig 2.

$$
R=S \times L
$$

OBSOLESCENCE RISK

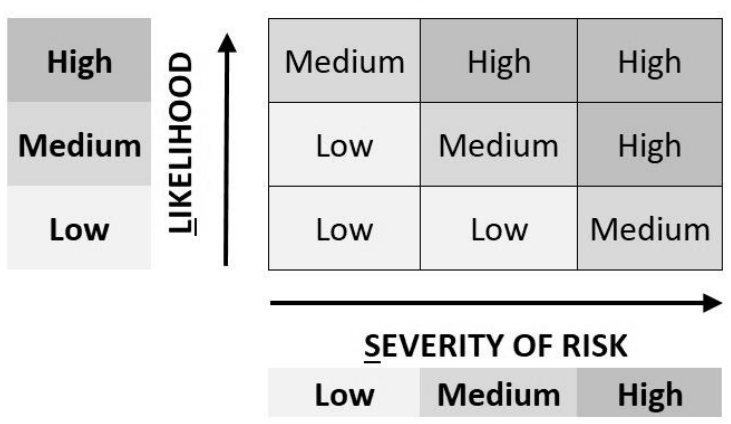

Figure 3: Obsolescence risk metric

Volume of Use [V]:

- Low - No widespread usage

- Medium - Widespread usage in a limited domain of applications

- High - Widespread usage in multiple domain of applications

Difficulty of Replacement [D]:

- Low - Easy to replace (TRL7 substitutes exist

- Medium - Moderately difficult to replace (3+years needed for TRL7 substitutes)

- High - Very difficult to replace (5+ years needed for TRL7 substitutes)

Obsolescence risk is thus defined in Eq. 2.

$\mathrm{R}=\mathrm{S} \times \mathrm{L}(2)$

Where the Likelihood $\mathrm{L}$ is defined in terms of the status of the substance in regulatory processes as follows:

Likelihood of Obsolescence [L]:

- Low - Fulfilling SVHC criteria, included in 'Substitute It Now' (SIN) list, under Community Rolling Action Plan (CoRAP) evaluation or other indication of obsolescence risk (eg specific Annex XVII restrictions not constituting a ban, but detailed conditions of use).

- Medium - Prioritized for inclusion to the Annex XIV, Candidate list

- High - Annex XIV, sunset date known; Annex $\mathrm{XVII}$ restriction banning a use, including for space

The overall risk metric is shown in Fig. 3.

14 ECSS-S-ST-00-01C - ECSS system Glossary of terms [2012 
The resulting actions for a given overall risk level are in turn determined by the level of risk acceptance for a given organization or project. We propose the following baseline for actions for the risk levels:

- Low - Tolerate, investigate

- Medium - Monitor, plan

- High - Mitigate, control

Continuous monitoring of the relevant regulatory evolutions, especially under REACH and CLP, and practical assessment of their impact on the future availability of space-qualified materials and processes are essential elements of the risk assessment and mitigation process. The Materials and Processes Technology Board of the European Space Components Coordination (ESCC ${ }^{15}$ MPTB) has been a key platform for space agencies and industry to collaborate on this important topic and pool resources.

\section{Substances Under Scrutiny}

A number of specific substances of interest to the space community that are under REACH regulatory scrutiny are highlighted below. This listing is not intended to be exhaustive, but rather an illustration of how different substances are being addressed in various REACH regulatory processes.

\section{Chromium trioxide (CAS: 1333-82-0)}

Overall obsolescence risk is High. Obsolescence risk parameters are: High Likelihood, Medium Volume of Use, Medium Difficulty of Replacement.

Chromium trioxide is a key enabling substance for manufacturing corrosion resistant coatings of metal alloys, with limited replacements available. It is included in Annex XIV, with an elapsed sunset date in 2017. Space industry supported ${ }^{16}$ an upstream Application for Authorisation (AfA) that was submitted by the 'CTACSub' consortium (Chromium Trioxide REACH Authorization Submission Consortium) in 2015. In 2016, the ECHA Committees for Risk Assess-

15 ESA, 'Charter of the European Space Components Coordination', [2018] 2 ESCC 1-19; See <https://spacecomponents.org/> accessed 13 May 2020.

16 See <https://echa.europa.eu/documents/10162/18074545/a4a _comment_665_1_attachment_en.pdf > accessed 13 May 2020. ment (RAC) and Socio-Economic Analysis (SEAC) recommended to grant the authorisation with a review period of 7 years under 'strict conditions'. In February 2019, this outcome was also initially supported by the REACH Committee of EU Member State representatives assisting the European Commission. However, due to a legal challenge before the Court of Justice of the European Union (CJEU) in another authorisation case (T-837/16), a resolution of the European Parliament of 27 March 2019 and changes in the European Commission, the outcome of the application is still pending today, and a new vote by the REACH Committee is expected to be required. In the meantime, downstream uses covered by the CTACSub AfA are continued to be allowed, in spite of the long expired sunset date.

The experience with the CTASub application has caused a number of important lessons learned and best practices being announced for future AfAs. It is clear that broad-scope upstream applications without a clear definition of the use, a detailed chemical safety assessment and analysis of alternatives will not be a feasible way forward, but rather more narrow applications with strict limitations and a substitution plan (where alternatives are available in general) are now expected.

Several actions are ongoing in European Space Agency, national space agencies and in the European space industry. Substitution of the substance varies on case-by-case basis depending on the alloy selected for coating. One of the main substances being used as a replacement is trivalent chromium, and the risks associated are explained in paragraph 5.5.

\section{Hydrazine (CAS: 302-01-2)}

Overall obsolescence risk is Medium. Obsolescence risk parameters are: Medium Likelihood, Medium Volume of Use, Medium Difficulty of Replacement.

Hydrazine has been included in the candidate list since 20 June 2011. Space industry, together with agencies, carried out dedicated REACH technical and legal assessments in the frame of the Hydrazine Task Force (HTF) established for this purpose. The resulting position paper was presented to the European Commission in October 2012. It advocates continued usage of hydrazine as a space vehicle propellant under automatic REACH authorisation exemption clauses, especially the 'use as fuels in closed systems' 
according to REACH Article 56 (4)(d), $2^{\text {nd }}$ alt. Currently the legal clarification by the European Commission is still pending.

In 2019, HTF has been re-activated in order to extend the exemption analysis to liquid bi-propellants MonoMethyl Hydrazine (MMH), Unsymmetrical DiMethyl Hydrazine (UDMH) and Dinitrogen Tetraoxide (NTO), and include the conclusions in the hydrazine position paper, together with some regulatory updates. The overall exemption position is not affected by this revision. The Revised Space Industry Position $2020^{17}$ was published by ASD-Eurospace on 15 April $2020^{18}$ and sent to the European Commission for its pending legal clarification.

\section{Lead metal (CAS: 7439-92-1)}

Overall obsolescence risk is High. Obsolescence risk parameters: Medium Likelihood, High Volume of Use, High Difficulty of Replacement.

Used for soldering (tin-lead) in electronic assemblies, as well as in copper and brass alloys and in some cases plating and mechanical lubrication (see Eurospace Position Paper ${ }^{19}$ for further application details). Lead metal was included in the candidate list for authorisation in 2018. Furthermore, OEL revision process for lead was initiated in 2019.

Several actions are ongoing in agencies and industry. Substitutes in soldering applications have been studied for many decades, but currently no replacements are available at a high TRL. More detailed reviews show the effects of REACH on electronics components in Europe $^{20}$.

\section{N-Methyl-2-pyrrolidone (CAS: 872-50-4)}

Overall obsolescence risk is High. Obsolescence risk parameters: High Likelihood, Medium Volume of Use, Medium Difficulty of Replacement.

N-Methyl-2-pyrrolidone (NMP) is used, for example, as a solvent in the production of the positive electrode within Li-ion batteries that are qualified for use on European space launchers and vehicles, and as a catalyst for adhesive formulation. NMP has been on the REACH candidate list since 20 June 2011. NMP was part of the $8^{\text {th }}$ recommendation of ECHA of 5 February 2018 for inclusion on Annex XIV (autho- rization path). The space industry has advocated against Annex XIV inclusion (see NMP Position ${ }^{21}$ ). Even though ECHA has recommended the substance for Annex XIV inclusion, a Regulatory Management Option Analysis (RMOA) by the European Commission with the collaboration of ECHA for three aprotic solvents (incl. NMP) concluded that targeted restrictions under REACH Annex XVII are most appropriate. Restrictions on use took effect on 9 May 2020. ECHA published a guideline document on compliance to the new restriction, focusing on handling, as well as biomonitoring of workers. ${ }^{22}$ Even though the substance was not included in Annex XIV, the regulatory activities caused at least one supplier to send a last-buy note for a family of products containing the substance in question.

\section{Chromium (III) oxide (CAS: 1308-38-9)}

Overall obsolescence risk is Low. Obsolescence risk parameters: Low Likelihood, Low Volume of Use, Low Difficulty of Replacement.

Chromium (III) oxide, trivalent chromium, is used in many substances that are being investigated as substitutes for chromium trioxide, hexavalent chromium. However, this substance is also being investigated for its toxicological properties. The investigation is performed in the frame of a CoRAP evaluation. ${ }^{23}$ Therefore, a risk of regrettable substitution exists, meaning that a hazardous substance is substituted with another one, which in turn is under obsolescence risk. The timelines of the evaluation are nondeterministic and difficult to estimate, which makes risk assessment particularly challenging.

17 See <https://eurospace.org/wp-content/uploads/2020/04/hydrazine -revised-reach-position-2020-final.pdf> accessed 13 May 2020.

18 See <https://eurospace.org/asd-eurospace-reinforces-reach -exemption-position-for-hydrazine-and-other-liquid-propellants/> accessed 13 May 2020.

19 See <https://p7665.phpnet.org/wp-content/uploads/2018/05/ Itffinalcommentonpb-20042018.pdf> accessed 13 May 2020.

20 Paavo Heiskanen, 'REACH update - Impact on availability of components for Space in Europe' (European Space Components Conference, Noordwijk, March 2019)

21 See <https://p7665.phpnet.org/wp-content/uploads/2018/05/es -nmp-spaceindustrycontribution.pdf> accessed 13 May 2020.

22 See <https://echa.europa.eu/fi/-/advice-on-how-to-comply-with -nmp-restriction> accessed 13 May 2020.

23 Latest status, <https://echa.europa.eu/information-on-chemicals/ evaluation/community-rolling-action-plan/corap-table/-/dislist/ details/0b0236e1820e1d0f> accessed 13 May 2020. 


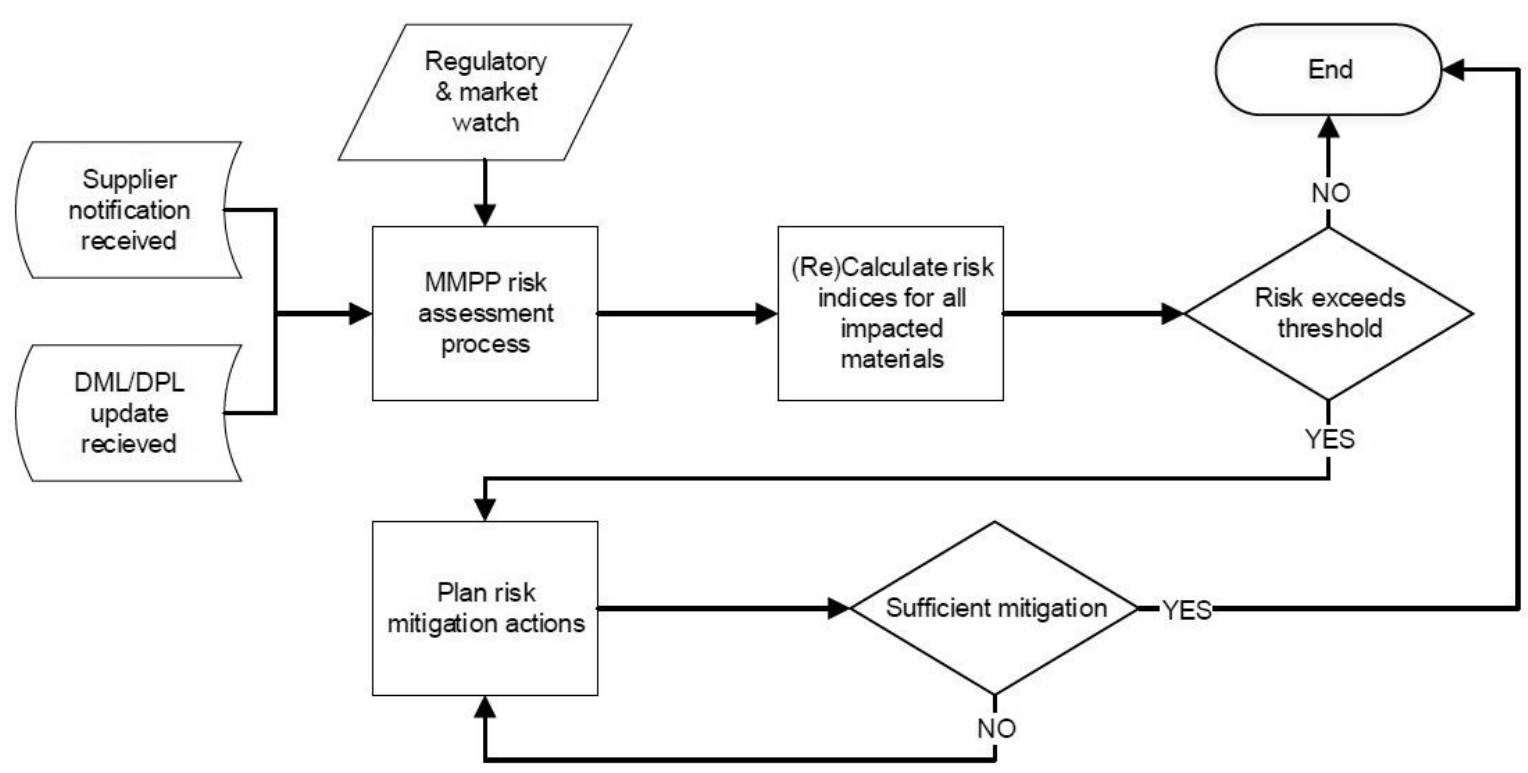

Figure 4: Obsolescence Risk Management Process

\section{Regulatory Obsolescence Risk Management}

As shown in previous paragraphs, health, safety and environmental regulations may create obsolescence risks for materials and processes that need to be mitigated. Any organisation that manufactures space hardware should generate an Obsolescence Management Plan (OMP) that defines the key elements on the organisation's approach to obsolescence. The ECSS handbook ${ }^{24}$ defines the key resources, approaches and concepts to obsolescence management.

We propose an example process, which is shown in Fig. 4. The risk assessment for materials, mechanical parts and processes (MMPP) takes input information from suppliers and regulatory market intelligence. In addition, updates to the Declared Materials List or Declared Process List (DML/DPL) ${ }^{25}$ of projects are continuously and proactively assessed in order to determine their exposure to different types of obsolescence. If a change is detected, the impact should be assessed, such as through using a methodology similar to the one introduced in paragraph 4 .

24 ECSS-Q-HB-70-23A - Materials, mechanical parts and processes obsolescence management handbook

25 ECSS-Q-ST-70C - Materials, mechanical parts and processes
If a risk metric value exceeds a predefined threshold, a set of actions are automatically initiated, starting a risk mitigation process. When the mitigation plan is in place to reduce the risk sufficiently, the plan is executed and the process ends. SVHCs contained in substances used for manufacturing is readily available information, and a key consideration for this type of process. This information can be acquired from Safety Data Sheets (SDS), which are obtained from the suppliers of the substances and mixtures.

Considering that projects in the space industry have a very high complexity, consisting of supply chains that can be up to ten layers deep and hundreds of organisations wide at the base, it is necessary that this process is executed in an automated fashion, ie an obsolescence management database is needed. It is also important to ensure that sub-suppliers have their own OMPs drafted and effective obsolescence management processes in place.

\section{Other Considerations}

\section{Responsibilities of article and complex object suppliers}

REACH (Article 33) defines an article as 'an object which during production is given a special shape, sur- 
face or design which determines its function to a greater degree than does its chemical composition'. Complex object, respectively, is a collection of articles as such joined or assembled. For example, the plated metal sheet used to cover an electronics assembly is an article, and the assembly itself is a complex object.

Substance manufacturers and mixture formulators have responsibilities to communicate safe use information, on certain hazardous substances present in their products, through SDSs to their customers. In addition, EU/EEA article and complex object producers and importers have the responsibility under REACH to communicate sufficient information, available to them, on candidate list substances in articles in the supply chain to allow safe use. The communication requirements are set out in REACH Article 33, and thus the corresponding document is often called a 'REACH Article 33 declaration', or a 'REACH declaration'. The candidate list substance concentration threshold triggering the reporting requirement is $0.1 \% \mathrm{w} / \mathrm{w}$ in the article supplied as such or as part of a complex object. This means that if the $0.1 \% \mathrm{w} / \mathrm{w}$ threshold is exceeded in a component article, the substance must be reported in the supply chain in the declaration. This was clarified in a judgment of the European Court of Justice of 10 September 2015 (case C-106/14) which ruled 'Once an Article, Always an Article', meaning that the concentrations in complex objects supplied cannot be calculated based on the overall weight of the object, but rather the concentration in each component article containing the substance ${ }^{26}$. This means - according to ECHA - that suppliers must now also report the component article(s) containing the substance, eg for solder joints. To support the aerospace and defence industries on how to fulfil the REACH Article 33 requirement under the aforementioned judgment, European Aeronautics, Space, Defence and Security Industries (ASD) has generated a guidance document ${ }^{27}$, which promotes the concept of aggregation of information to higher levels of very complex objects (sub-/assemblies).

Another requirement that is becoming more relevant to the space industry is the notification to be directed to ECHA if a substance under an approved upstream AfA is being used. This REACH Article 66 notification will become relevant for cases such as when the authorisation decision for chromium trioxide is formally adopted by the European Commission and is made known to the downstream users (through the authorisation holder or publication on the internet). It is important to note that this notification duty only applies to the company acting as a chemical user (eg a company carrying out surface treatment operations using chromium trioxide); it does not apply to assemblers and suppliers of already surface-treated hardware (they may be subject to REACH Article 33).

\section{SCIP Database}

During the course of 2019, ECHA has published its first detailed plans on how the SCIP database under the revised Waste Framework Directive is going to be implemented. Currently, an ECHA SCIP 'prototype' is available for testing purposes. SCIP notifications by industry shall be possible from the end of October 2020.

The space industry impact of this new SCIP notification and database requirement is currently still being determined. A dedicated WFD Task Force has been created to this end ${ }^{28}$. As a result of this task force, Eurospace has published a position paper ${ }^{29}$ on 9 September 2019, advocating that SCIP notification should not apply to equipment designed to be sent into space and related means of transport (launch vehicles and spacecraft, such as satellites for telecommunication, navigation or space exploration) which does not result in waste on the EU territory. In the absence of an authoritative exemption for such equipment, the task force has now started the development of sectoral guidelines for compliance with SCIP notification tailored to its specific case.

ECHA is proceeding with the SCIP database development based on its detailed information requirements ${ }^{30}$. This means that EU (and potentially EEA)

26 The judgment is available at <http://curia.europa.eu/juris/ document/document.jsf?text $=\&$ docid $=167286 \&$ pagelndex $=0$ $\&$ doclang $=$ EN\&mode $=$ Ist $\&$ dir $=\&$ occ $=$ first $\&$ part $=1 \& \mathrm{cid}=754885>$ accessed 13 May 2020.

27 'Sectoral Guidance for Substances in Articles under REACH', [2017] 1 ASD 1-57

28 Eurospace: New Space Industry Task Force to address the EU's revised Waste Framework Directive, 8 August 2019

29 See <https://eurospace.org/space-industry-position-regarding -article-9-of-the-revised-waste-framework-directive-2008-98-ec/> (accessed 13 May 2020)

$30 E C H A$, Detailed information requirements for the SCIP database, September 2019, available at < https://echa.europa.eu/documents/ 10162/28213971/scip_information_requirements_en.pdf $>$ accessed 15 November 2019. 
suppliers of articles on each level of the supply chain need to report the required information on all their products placed on the market, provided that they contain candidate list substances above $0.1 \% \mathrm{w} / \mathrm{w}$ in any component article. The information requirements and envisioned public access to data, published by ECHA, seem to go above and beyond the requirements that are specifically listed in the legal text and REACH Article 33, causing concerns on Intellectual Property rights and the feasibility for industry to fulfil the responsibilities. A legal analysis ${ }^{31}$ of the approach has been performed.

\section{UK withdrawal from the EU}

The withdrawal of the United Kingdom from the European Union has caused uncertainty for the space industry. After several deferrals, the UK left the European Union on 31 January 2020. This means that the UK is no longer an EU Member State starting from 1 February 2020. However, based on the Withdrawal Agreement concluded between the EU and the UK, the EU REACH Regulation and all other EU law will continue to apply in the UK as they currently do, for the time of the so-called transition period lasting at least until 31 December 2020. During this transition period it will be 'business as usual' for companies in both the EU and the UK. The transition period will be used by the EU and the UK to agree on a new partnership for the future.

When considering the industry implications for a non-EU entity of the REACH regulation, it needs to be recalled that the regulation is also applied in the non-EU Member States of the EEA, ie Norway, Ice-

31 Tim Becker, 'The SCIP Database under Directive (EU) 2018/851' (2019) 2 StoffR 156

32 See <https://www.efta.int/eea-lex/32006R1907> accessed 20 April 2020

33 See <https://www.anmeldestelle.admin.ch/chem/en/home/ themen/recht-wegleitungen/stand-der-harmonisierung-mit -internationalen-vorschriften.html> accessed 15 April 2020.

34 Revised Protocol on Ireland and Northern Ireland included in the Withdrawal Agreement, 17 October 2019; See <https://ec.europa .eu/commission/publications/revised-protocol-ireland-and -northern-ireland-included-withdrawal-agreement_en $>$ accessed 29 April 2020; European Commission, Notice to stakeholders, Withdrawal of the United Kingdom and the EU rules in the field of chemicals regulation under REACH, 30 March 2020; see <https://ec.europa.eu/info/sites/info/files/notice_to_stakeholders _brexit_reach.pdf $>$ accessed 29 April 2020.

35 See <http://www.legislation.gov.uk/ukpga/2018/16/section/15A> accessed 19 May 2020. land and Liechtenstein ${ }^{32}$. Switzerland, despite not being an EU/EEA Member State, pursues an independent harmonisation of its national chemicals legislation with EU REACH ${ }^{33}$.

The EU-UK negotiations on the new partnership will also determine if a separate chemical safety regulation ('UK REACH') will apply after the end of the transition period. One possible scenario under such a 'UK REACH' is that the UK would have its own central regulatory authority, the Health and Safety Executive (HSE). The regulatory framework may be based on the EU REACH system, with similar processes in place for authorisation, supply chain communication, etc. However, it is important to note that a change of authorities in charge away from ECHA, EU institutions and Member States to UK-only decisionmakers may result in a regulatory divergence in the future, ie a substance may be under a REACH authorisation or restriction in the EU/EEA, but not in the UK or vice versa. This will create added complexity on the supply chain considerations that go across the two regulatory regimes. Furthermore, it would imply that manufacturers, formulators and suppliers that operate in both systems will need to comply with both regimes separately, one for EU and one for the UK. In respect of Northern Ireland, EU REACH would continue to apply even longer than in the remaining UK, subject to the Protocol on Ireland/Northern Ireland ${ }^{34}$.

An alternative scenario is that the transition period would be extended beyond 2020 for up to two years, ie until 31 December 2022 at the latest. In this case EU REACH would continue to apply in the entire UK during such extended period. Such extension would need to be agreed by a decision of the UK-EU Joint Committee before 1 July 2020. However, this would require the UK domestic ban on extending transition under Section 15A of the EU (Withdrawal) Act 2018 currently in force ${ }^{35}$ to be repealed. A possible factor determining this could be the COVID-19 disruptions.

\section{Conclusions and Outlook}

Health, safety and environmental regulations are impacting production for the EU/EEA market at every level of the value chain, particularly in industrial sectors with complex supply chains and long project durations, such as the space industry. It was concluded 
early on ${ }^{36}$ that the whole industry needs to respond to the requirements and issues generated by the increasing regulations. Potentially any hazardous substance fulfilling SVHC criteria or presenting an unacceptable risk used in manufacturing may become obsolete in the span of five to six years from the first indication of regulatory activity.

This timescale is incompatible with space project durations and mandates a strong and proactive obsolescence management programme for all industrial actors. Due to the vast number of materials and processes used to manufacture products, that consist of tens of thousands of parts, automated systems are required to manage the obsolescence risk, in addition to company and sector level working groups operating on a continuous basis (such as the MPTB). These systems rely on timely and accurate information on the substances under regulatory scrutiny. This task is not made easier by recent developments in the regulatory framework. For example, the candidate list now contains several substance entries that are not identified by CAS numbers. A prominent example is a recent entry 'perfluorobutane sulfonic acid (PFBS) and its salts', where the industry estimates well over one thousand CAS numbers to be included under one substance name. The entry includes all current forms of the substance, as well as the ones that will be invented in the future. While this approach is, according to the regulators, chemically and toxicologically sound, it raises questions on the feasibility of implementation by industry, where supply chain communication is based on clear numerical identifiers. This problem is exacerbated by the growing number of SMEs and other new space companies that operate on small budgets and with a limited experience. It is important to remember that every entry in the candidate list also generates requirements with respect to Article 33 declarations, and in the future for SCIP notifications (unless an exclusion or exemption applies).

The processing of the upstream CTACSub AfA for chromium trioxide in the EU decision-making process has taken several years and is still ongoing, and meanwhile end users of this critical enabling substance for the space industry are working based on the assumption that the AfA will be approved. However, the authorisation will only be granted for a limited duration, and the start date is typically counted from the original sunset date, not from the date of adoption of the authorisation decision (with some exceptions). Therefore the upcoming time period, before a re-authorisation will become necessary, may also be shrinking as long as the decision is still pending. It should be recalled that a primary substitute (based on trivalent chromium) is also subject to a possible obsolescence risk, in an assessment process (REACH substance evaluation) where the timeline for future regulatory activities is neither known nor predictable. This issue of regrettable substitution is very prominent for energetic materials, nominally propellants and explosives. These are very widely used in the space domain, and are often by their chemical nature volatile and hazardous. This generates an obvious risk for all space projects.

Finally, it should be noted that the regulations discussed are highly desirable and ambitious, contributing to human health and a safer environment. The success of the RoHS Directive and the REACH Regulation can be seen by the number of countries globally that have followed their examples. This is also good news for the competitiveness of the European industry. As European companies are substituting their hazardous chemicals and qualifying safer alternatives already, they will have a competitive advantage on other markets when these substances come under regulatory scrutiny overseas.

Overall, despite the issues listed in this paper, it has to be acknowledged that REACH is contributing towards a better protection of human health and the environment. It incentivizes research and development in innovative substitution of hazardous chemicals and enables sustainable global competitiveness for European industry for years to come. Within the European space industry, it has been observed that companies have engaged in deep technical collaboration between competitors rather than using the regulation as a competitive advantage. Key business enablers have been proactive obsolescence risk management, communication, and collaboration.

Acknowledgements: The authors would like to thank all the members of the MPTB for their continued support and collaboration on the field of obsolescence risk management for space industry. With-

36 Thomas Rohr, 'Impact of REACH legislation on European space programs', International workshop on environment and alternative energy

ESAC, 12 November 2015, See: <https://tdglobal.ksc.nasa.gov/ servlet/sm.web.Fetch/Rohr_- 151112_REACH_presentation_1.0.pdf ? rhid $=1000 \&$ did $=916169 \&$ type $=$ released $>$ accessed 14 May 2019. 
out their willingness to share information and co-operate on refining the best practices and ongoing issues this paper would not have been possible.

Disclaimer: The information in this article reflects the opinion of the authors. It is not considered a requirement nor a comprehensive treatment of the subject matter, and any action to the described issues are subject to project or programme decision. The article is intended for information only and whilst it is provided in utmost good faith and has been based on the best information currently available and adequate technical standards, is to be relied upon at the reader's own risk. No representations or warranties are made with regards to its completeness, or accuracy and no liability will be accepted by the European Space Agency, REACHLaw Ltd and the authors for damages of any nature whatsoever resulting from the use of or reliance on the information. 